\title{
The application of shotgun metagenomics to the diagnosis of granulomatous amoebic encephalitis due to Balamuthia mandrillaris: a case report
}

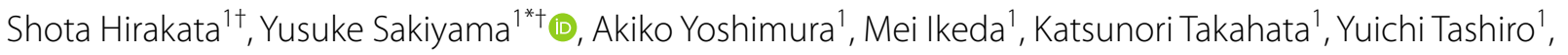
Michiyoshi Yoshimura' ', Hitoshi Arata ${ }^{1}$, Hajime Yonezawa², Mari Kirishima ${ }^{3}$, Michiyo Higashi ${ }^{3}$, Miho Hatanaka ${ }^{4}$, Takuro Kanekura ${ }^{4}$, Kenji Yagita ${ }^{5}$, Eiji Matsuura ${ }^{1}$ and Hiroshi Takashima ${ }^{1}$

\begin{abstract}
Background: Granulomatous amoebic encephalitis (GAE) is an infrequent and fatal infectious disease worldwide. Antemortem diagnosis in this condition is very difficult because clinical manifestations and neuroimaging are nonspecific.

Case presentation: A 60-year-old Japanese woman was admitted with a chief complaint of left homonymous hemianopsia. Brain-MRI showed extensive necrotizing lesions enhanced by gadolinium, in the right frontal lobe, right occipital lobe, and left parietal lobe. Epithelioid granulomas of unknown etiology were found in the biopsied brain specimens. Shotgun metagenomic sequencing using a next-generation sequencer detected DNA fragments of Balamuthia mandrillaris in the tissue specimens. The diagnosis of granulomatous amoebic encephalitis was confirmed using an amoeba-specific polymerase chain reaction and immunostaining on the biopsied tissues.
\end{abstract}

Conclusions: Shotgun metagenomics is useful for the diagnosis of central nervous system infections such as GAE wherein the pathogens are difficult to identify.

Keywords: Granulomatous amoebic encephalitis, Balamuthia mandrillaris infection, Necrotizing encephalitis, Nextgeneration sequencer, Shotgun metagenomics

\section{Background}

Granulomatous Amoebic Encephalitis (GAE), caused by the free-living amoeba Balamuthia mandrillaris, was first reported in humans in 1990 [1]. Currently, nearly 200 cases have been reported worldwide [2-4]. Clinical presentation and neuroimaging in this condition

\footnotetext{
*Correspondence: ysksakiyama@gmail.com

${ }^{\dagger}$ Shota Hirakata and Yusuke Sakiyama contributed equally to this work.

1 Department of Neurology and Geriatrics, Kagoshima University

Graduate School of Medical and Dental Sciences, 8-35-1 Sakuragaoka,

Kagoshima City, Kagoshima 890-8520, Japan

Full list of author information is available at the end of the article
}

are nonspecific, and $B$. mandrillaris is rarely detected in common bacteriological tests for cerebrospinal fluid (CSF), making the diagnosis of GAE extremely difficult [2-5]. Herein, we report a case in which B. mandrillaris was identified by unbiased metagenomic sequencing of brain biopsy specimens and antemortem diagnosis of GAE was made.

\section{Case presentation}

A 60-year-old Japanese woman was admitted to our hospital because of visual field loss and unsteady gait. The patient spent time gardening as a hobby and was 
on infliximab and methotrexate therapy for rheumatoid arthritis. Two months before admission, her left visual field loss had steadily progressed, and she felt low-grade fever and general malaise. A high signal in the right occipital lobe and the left parietal lobe was detected in the MRI fluid-attenuated inversion recovery (FLAIR), and the lesions were enhanced by gadolinium (Fig. 1AD). Brain biopsy of the right occipital lobe lesion demonstrated granulomatous inflammation, but no pathogenic micro-organisms were detected using either microscopic examination or culture. A diagnosis of neurosarcoidosis was made by the neurologists based on the brain biopsy findings.

The symptom of unsteady gait was temporarily relieved by intravenous methylprednisolone (IVMP); although, it worsened soon after discharge, followed by readmission to our department. At the time of readmission, there were no notable findings in the chest and abdomen and no uveitis was noted. A brown spot with a scab, about 10 millimeters in diameter, was observed inside the left upper arm. Neurologically, left lower visual field and mild weakness in the distal muscles of the upper limbs were

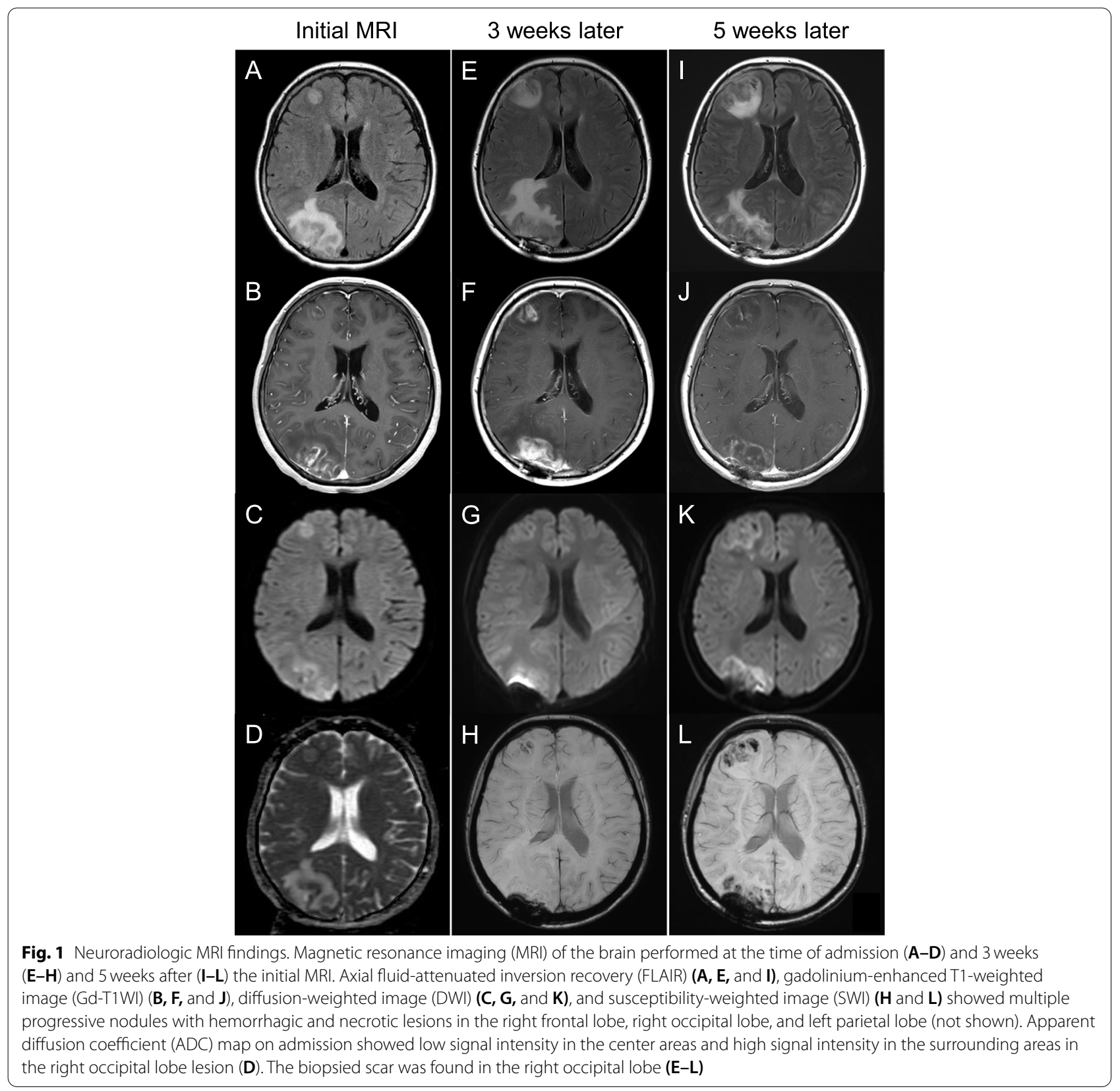


observed. Deep tendon reflexes and muscle tonus in the upper and lower limbs were normal, and there was no ataxia or sensory impairment. Analysis of blood samples showed no abnormal findings in the blood cell count or the coagulation system. Serum angiotensin-converting enzyme II activity was $12.9 \mathrm{U} / \mathrm{L}$ and lysozyme level was $6.2 \mu \mathrm{g} / \mathrm{mL}$. Antinuclear and antineutrophil cytoplasmic antibodies were negative. Specific antibodies for aquaporin 4 and myelin oligodendroglia were also negative. Tumor markers were not elevated. Antiparasitic antibodies and blood cultures were also negative. The CSF collected 1 week after brain biopsy was colorless and transparent, and the initial cerebrospinal pressure was $12 \mathrm{cmH}_{2} \mathrm{O}$. The CSF cell count was measured to be elevated at $43 / \mu \mathrm{L}$ (neutrophil $0 / \mu \mathrm{L}$, lymphocyte $38 / \mu \mathrm{L}$, and monocyte $5 / \mu \mathrm{L}$ ), in addition to the increased protein levels at $77.8 \mathrm{mg} / \mathrm{dL}$. Cerebrospinal fluid sugar level was $86 \mathrm{mg} / \mathrm{dL}$, while the blood glucose level was detected at $192 \mathrm{mg} / \mathrm{dL}$. The CSF/serum ratio of albumin (Q-albumin) was elevated at 14.2 (CSF albumin: $57 \mathrm{mg} / \mathrm{dL}$, serum albumin: $4010 \mathrm{mg} / \mathrm{dL}$ ). Gram staining did not detect any bacteria, and common bacteria and acid-fast bacteria were not cultured from the CSF. Cryptococcus neoformans antigen, Aspergillus antigen, Toxoplasma antibody, and Human Polyomavirus 2 DNA were all negative. Transthoracic echocardiography showed no obvious thrombi or warts. Skin biopsy performed on the brown spot on the left upper arm showed dense lymphoplasmacytic inflammation distributing from the upper dermis to the subcutaneous adipose tissue with a few epithelioid cell granulomas. Contrast-enhanced computed tomography $(\mathrm{CT})$ of the region from the cervix through the pelvis revealed no neoplastic lesions or bilateral hilar lymphadenopathy, and gallium scintigraphy did not reveal any abnormal accumulation.

Brain MRI after IVMP showed enlarged lesions with internal necrosis (Fig. 1E-H). At 16 days after admission, ceftriaxone $(2 \mathrm{~g} /$ day $)$, acyclovir $(1.2 \mathrm{~g} /$ day $)$, fosfluconazole $(0.4 \mathrm{~g} /$ day $)$ sulfamethoxazole $(1.2 \mathrm{~g} /$ day $)$, and trimethoprim $(0.24 \mathrm{~g} /$ day $)$ were initiated along with the second IVMP to cover both autoimmune encephalitis and infectious meningitis caused by bacteria, herpes virus, Cryptococcus neoformans, and parasites. However, the treatment response was poor, and new lesions appeared in the medulla oblongata (data not shown). Because the patient suddenly fell into a coma on day 21 after admission, intratracheal intubation was performed and ventilator management was started. Brain MRI revealed progressed necrosis and edema (Fig. 1I-L). A second brain biopsy with needle aspiration from reddishbrown tissue revealed necrotic tissue which was considered to be reflecting brain abscess, but no pathogenic micro-organisms were detected using either microscopic examination or culture. Furthermore, neither tumor nor granulomas were observed in the specimen.

In order to identify the causative pathogenic microorganism, the initial brain biopsy specimens were subjected to shotgun metagenomic sequencing using a next-generation sequencer (NGS). The DNA extracted from the brain specimens was comprehensively analyzed using Illumina sequencer (MiSeq ${ }^{\circledR}$ ) and compared with the sequences registered in the database through the BLASTN analysis. As a result, 129 DNA fragments homologous to $B$. mandrillaris were identified from the 9 million DNA fragments derived from the brain specimens (Fig. 2A). The PCR analysis for the mitochondrial rRNA of B. mandrillaris [6] revealed bands of the PCR product in the patient's brain and skin biopsy specimens (Fig. 2B, Additional file 1: Fig. S1). The pathogen detected from the present case was presumed to be a strain with a high degree of similarity to B. mandrillaris V039 based on phylogenetic analysis using MEGA X [7] (Fig. 2C). Based on these findings, histopathological specimens of the brain tissue (Fig. 3A-D) and skin biopsy (Fig. 3E) were re-evaluated microscopically. Brain biopsy of the right occipital lobe lesion demonstrated granulomatous inflammation, containing epithelioid granuloma with palisading necrosis (Fig. 3A) and multiple epithelioid granulomas without necrosis (Fig. 3B). Moreover, multinucleated giant cells containing amoebic trophozoites were seen on hematoxylin and eosin (H\&E) stain (Fig. 3C) and these trophozoites were confirmed immunohistochemically with anti- $B$. mandrillaris rabbit antibody (Fig. 3D). Skin biopsy also showed a few amoebic trophozoites (Fig. 3E) which were confirmed by immunohistochemical staining. Thus, the patient was diagnosed with GAE caused by $B$. mandrillaris. The treatments with azithromycin $(0.5 \mathrm{~g} /$ day $)$, sulfamethoxazole $(1.2 \mathrm{~g} /$ day $)$, trimethoprim $(0.24 \mathrm{~g} /$ day $)$, and fosfluconazole $(0.2 \mathrm{~g} /$ day) was initiated; however, the patient died 44 days after admission.

\section{Discussion and conclusion}

It is extremely difficult to make a clinical diagnosis of GAE while the patient is still alive. Of the 18 cases of GAE in Japan including the present case, 16 cases were diagnosed at autopsy, and only one case was successfully treated $[3,8]$. The reason for the difficulty in GAE diagnosis is the rapid disease progression and a lack of specific clinical symptoms $[2,5]$. Moreover, the trophozoites and cysts of $B$. mandrillaris are difficult to detect in CSF [2-5] and rarely grow on agar plates inoculated with mammalian cell cultures used to isolate $B$. mandrillaris [1, 2]. Brain lesions associated with GAE can be visualized as occupied lesions with hemorrhage and necrosis on brain CT and MRI, although these are not 


\section{A. Shotgun metagenomic sequencing data}

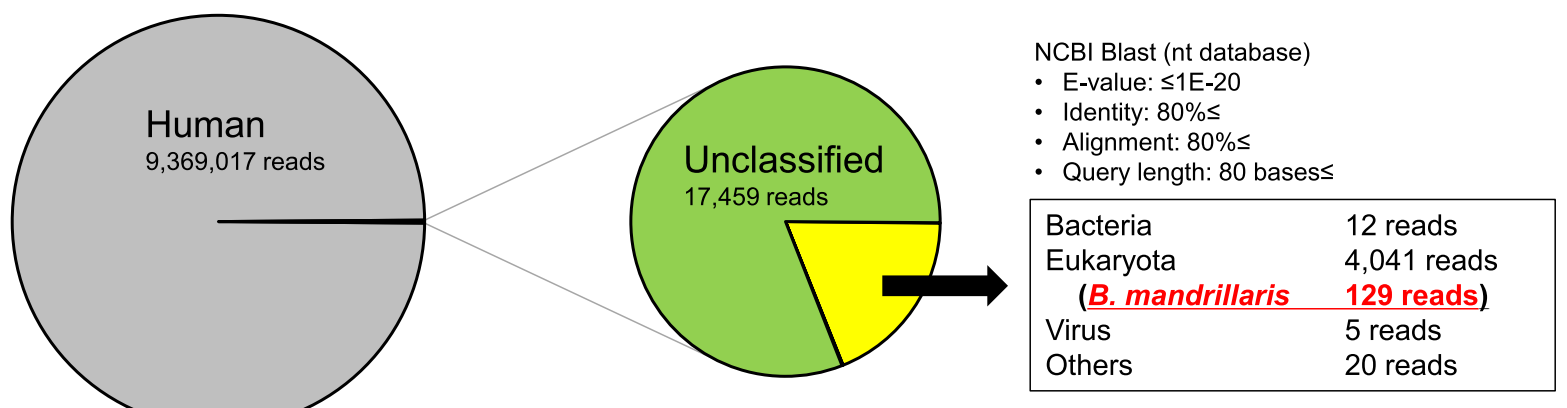

\section{B. PCR using B. Mandrillaris specific primer}

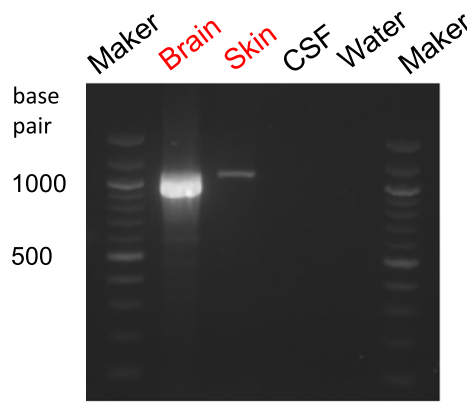

5'Balspec16S:CGCA TGTATGAAGAAGAC CA

3'Balspec16S:TTACC TATATAATTGTCGATA CCA

Product size: 1075 base pair

\section{Neighbor-joining tree} (mitochondrial rRNA gene)

${ }^{89}$ Balamuthia mandrillaris V039 present case

43 Balamuthia mandrillaris 2046-1

Balamuthia mandrillaris OK1

64 Balamuthia mandrillaris RP5

Balamuthia mandrillaris SAM

Balamuthia mandrillaris GAM-19

Balamuthia mandrillaris V188

Balamuthia mandrillaris V451

${ }_{88}$ Balamuthia mandrillaris $\mathrm{BeN}$

Fig. 2 Result of metagenomic NGS, PCR and phylogenetic analysis. A, Analysis of metagenomic next-generation sequencing (NGS) results from a patient. The total reads distribution is shown on the left and the distribution of reads without the human genome is shown on the right. Genomic DNA was extracted from the biopsied brain samples using an extraction kit (QIAGEN, Tokyo, Japan). Shotgun metagenomic sequencing was performed using the MiSeq ${ }^{\circledR}$ NGS system (NextraXT prep kit ${ }^{\circledR}$, Illumina) and a paired-end sequencing approach. For the identification of non-human DNA, the CLC Genomics Workbench was first used to query a human genome database (UCSC: hg38) and a human mRNA database (RefSeq release 54) and eliminate most human sequences. The remaining unmapped reads were subsequently analyzed by Nucleotide BLAST against the nt database from NCBI. A total of 129 reads showed a striking homology to B. mandrillaris under conditions of $\mathrm{E}$-value $<1 \mathrm{e}^{-20}$, hit length $>80$, and identity $>80 \%$. B. Polymerase chain reaction analysis using species-specific primers revealed a positive reaction for $B$. mandrillaris in the brain and skin samples from the patient and a negative reaction for B. mandrillaris in the CSF from the patient and the water control. $C$, Phylogenetic analysis of $B$. mandrillaris mitochondrial small subunit rRNA gene was performed based on the neighbor-joining method using MEGA X software. The percentage trees in which the associated taxa cluster together in the bootstrap test (1000 replicates) are shown next to the branches. Scale bars indicate the genetic distance

disease-specific findings $[9,10]$. Elevated antibody titers to B. mandrillaris are useful for diagnosis; however, these antibody tests are not commercially available. In addition, if the clinician does not suspect GAE, there is no opportunity to evaluate antibody titers. For these reasons, a biopsy of brain lesions is crucial for the diagnosis of GAE. Similar to the findings from brain imaging, the lesion exhibits hemorrhagic necrosis, chronic inflammatory infiltrates with sparse multinucleated giant cells, and diffuse lymphocytes and plasma cells [1]. Even if some amoeba trophozoites and cysts are present in the lesion, their identification may be challenging due to a general lack of familiarity with their morphology [11]. A background of extreme inflammation and necrosis can also risk masking the presence of the amoeba on routine histopathologic examination, such as in the present case.

The shotgun metagenomic sequencing using NGS has been demonstrated to be useful in the diagnosis of CNS infections that are difficult to diagnose [12-16]. Infectious and non-infectious CNS diseases are difficult to distinguish based on symptomology alone, and effective tests for rare pathogenic micro-organisms have not been established [12, 14]. Conventional diagnostic methods for CNS infections require several tests, including smear, culture, nucleic acid amplification, and serological tests, as well as a large number of CSF samples [16]. 


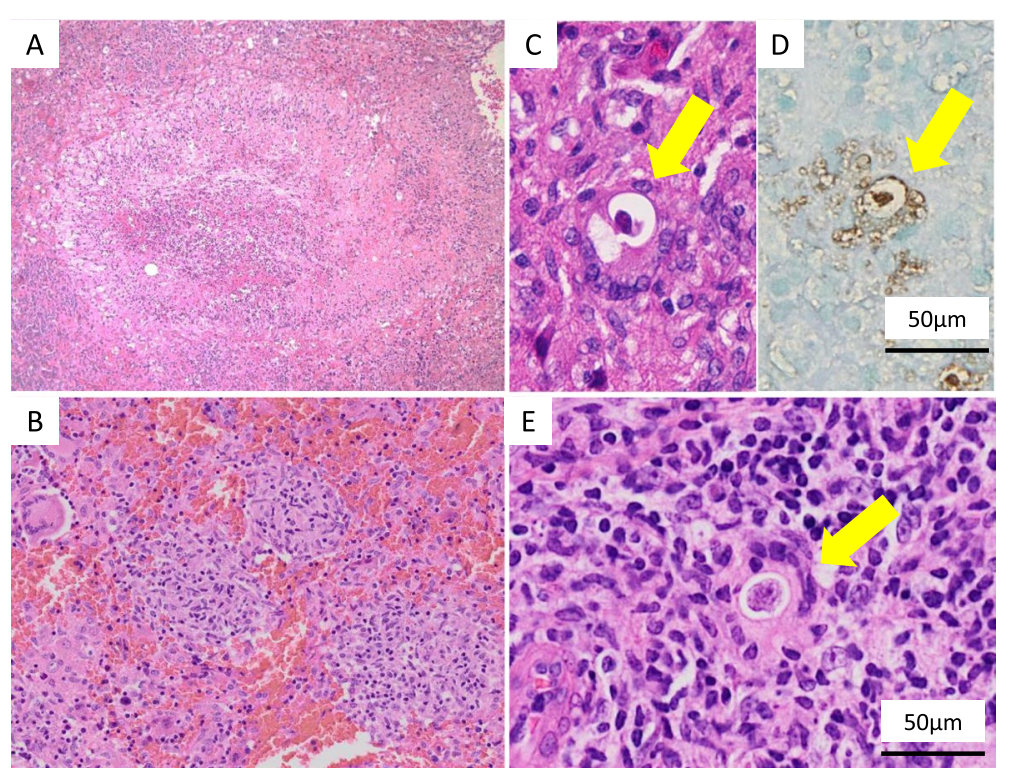

Fig. 3 Pathological findings of brain and skin biopsy samples. Histopathological findings of the brain biopsy (A, B, C and $\mathbf{D})$, and the skin biopsy (E). A, hematoxylin and eosin (H\&E) stained section showing necrotic tissue surrounded by palisading epithelioid histiocytes, forming epithelioid granuloma with necrosis (original magnification $\times 100$ ). B, Multiple epithelioid granulomas without necrosis were also seen (H\&E stain, original magnification $\times 200$ ). $\mathbf{C}$ and $\mathbf{D}$, Multinucleated giant cell containing an amoebic trophozoite $(\mathbf{C}, \mathbf{H} \& \mathbf{E}$ stain, highlighted by yellow arrow) was detected, which was confirmed by immunohistochemical staining with anti-B. mandrillaris rabbit antibody (D, highlighted by yellow arrow) (original magnification $\times 400$ ). E, Skin biopsy also showed multinucleated giant cells containing amoebic trophozoite (H\&E stain, highlighted by yellow arrow, original magnification $\times 400$ )

The shotgun metagenomic sequencing is an innovative diagnostic tool because it can facilitate a comprehensive evaluation of a variety of pathogenic microorganisms in a small amount of specimen using only a single assay [17]. In a study evaluating the efficacy of NGS for CNS infections of unknown etiology, 7 out of 204 cases were identified by metagenomic sequencing alone, contributing to the selection of effective treatments [14]. If this present case had undergone shotgun metagenomic sequencing at the time of the first brain biopsy, the patient could have been given more adequate treatment against the amoebae.

In conclusion, we made a diagnosis of GAE due to B. mandrillaris infection using shotgun metagenomic sequencing of biopsied brain specimen while the patient was still alive. Early diagnosis of amoebic encephalitis remains a challenge, and several cases may escape detection. We recommend that unbiased metagenomic sequencing of the affected tissues be performed in patients with CNS infections that are difficult to diagnose and treat, including amoebic encephalitis.

\section{Abbreviations}

GAE: Granulomatous amoebic encephalitis; CSF: Cerebrospinal fluid; MRI: Magnetic resonance imaging; IVMP: Intravenous methylprednisolone; NGS: Next-generation sequencer.

\section{Supplementary Information}

The online version contains supplementary material available at https://doi. org/10.1186/s12883-021-02418-y.

Additional file 1: Supplementary Figure S1. Unprocessed original gel/ blot images of polymerase chain reaction analysis using species-specific primers for B. mandrillaris.

\section{Acknowledgments}

The authors would like to thank Linc Science (https://www.lincscience.com) for the English language review.

\section{Authors' contributions}

SH wrote the initial draft of the manuscript. YS wrote the manuscript and performed the genetic examination. EM and $\mathrm{HT}$ revised the manuscript for important intellectual content. $\mathrm{HY}$ performed the brain biopsy. $\mathrm{MK}, \mathrm{MH} 1$, $\mathrm{MH} 2$, TK, and $\mathrm{KY}$ analyzed the pathological findings. AY performed the genetic examination. MI, KT, YT, MY, and HA participated in clinical data collection including medical history, neurological findings, hematological examination, electrophysiological evaluation, and treatment. All authors read and approved the final manuscript.

\section{Funding}

This work was supported by grants from Nervous and Mental Disorders and Research Committee for Applying Health and Technology of the Ministry of Health, Welfare and Labour, Japan. HT revised the manuscript for important intellectual content.

This work was also supported by JSPS KAKENHI Grant Number JP19K17013. YS wrote the initial draft of the manuscript and performed the genetic examination. 
Availability of data and materials

Not applicable.

\section{Declarations}

\section{Ethics approval and consent to participate}

The Institutional Review Board of Kagoshima University approved this study.

\section{Consent for publication}

Written informed consent was obtained from the patient's next of kin for publication of this case report and any accompanying images. A copy of the written consent is available for review by the Editor-in-Chief of this journal.

\section{Competing interests}

The authors declare that they have no competing interests.

\section{Author details}

${ }^{1}$ Department of Neurology and Geriatrics, Kagoshima University Graduate School of Medical and Dental Sciences, 8-35-1 Sakuragaoka, Kagoshima City, Kagoshima 890-8520, Japan. ${ }^{2}$ Department of Neurosurgery, Kagoshima University, 8-35-1 Sakuragaoka, Kagoshima City, Kagoshima 890-8520, Japan. ${ }^{3}$ Department of Pathology, Kagoshima University, 8-35-1 Sakuragaoka, Kagoshima City, Kagoshima 890-8520, Japan. ${ }^{4}$ Department of Dermatology, Kagoshima University, 8-35-1 Sakuragaoka, Kagoshima City, Kagoshima 890-8520, Japan. ${ }^{5}$ Department of Parasitology, National Institute of Infectious Diseases, 1-23-1, Toyama, Shinjuku-ku, Tokyo 162-8640, Japan.

\section{Received: 30 March 2021 Accepted: 29 September 2021}

Published online: 09 October 2021

\section{References}

1. Visvesvara GS, Martinez AJ, Schuster FL, Leitch GJ, Wallace SV, Sawyer TK, et al. Leptomyxid ameba, a new agent of amebic meningoencephalitis in humans and animals. J Clin Microbiol. 1990;28:2750-6.

2. Cope JR, Landa J, Nethercut H, Collier SA, Glaser C, Moser M, et al. The epidemiology and clinical features of Balamuthia mandrillaris disease in the United States, 1974-2016. Clin Infect Dis. 2019;68(1):1815-22.

3. Hara T, Yagita K, Sugita Y. Pathogenic free-living amoebic encephalitis in Japan. Neuropathology. 2019;39:251-8.

4. Schuster FL, Visvesvara GS. Free-living amoebae as opportunistic and non-opportunistic pathogens of humans and animals. Int J Parasitol. 2004;34:1001-27.

5. Ong TYY, Khan NA, Siddiqui R. Brain-eating amoebae: predilection sites in the brain and disease outcome. J Clin Microbiol. 2017;55:1989-97.
6. Booton GC, Carmichael JR, Visvesvara GS, Byers TJ, Fuerst PA. Identification of Balamuthia mandrillaris by PCR assay using the mitochondrial $16 \mathrm{~S}$ rRNA gene as a target. J Clin Microbiol. 2003;41(1):453-5.

7. Kumar S, Stecher G, Li M, Knyaz C, Tamura K. MEGA X: Molecular evolutionary genetics analysis across computing platforms. Mol Biol Evol. 2018;35(6):1547-9.

8. Kato H, Mitake S, Yuasa H, Hayashi S, Hara T, Matsukawa N. Successful treatment of granulomatous amoebic encephalitis with combination antimicrobial therapy. Intern Med. 2013;52(17):1977-81. https://doi.org/ 10.2169/internalmedicine.52.0299.

9. Singh P, Kochhar R, Vashishta RK, Khandelwal N, Prabhakar S, Mohindra S, et al. Amebic meningoencephalitis: Spectrum of imaging findings. Am J Neuroradiol. 2006;27:1217-21.

10. Ghosh PS, Ghosh D, Loddenkemper T, Prayson RA, Tekautz T, Sriram CS, et al. Necrotizing granulomatous meningoencephalitis due to Balamuthia in an immunocompetent child. Neurology. 2011;77(8):801-2.

11. Yagi S, Booton GC, Visvesvara GS, Schuster FL. Detection of Balamuthia mitochondrial 16S rRNA gene DNA in clinical specimens by PCR. J Clin Microbiol. 2005;43:3192-7.

12. Wilson MR, Shanbhag NM, Reid MJ, Singhal NS, Gelfand JM, Sample HA et al. Diagnosing Balamuthia mandrillaris encephalitis with metagenomic deep sequencing. Ann Neurol. 2015;78:722-30.13.

13. Sakiyama Y, Kanda N, Higuchi Y, Yoshimura M, Wakaguri H, Takata Y, et al New type of encephalomyelitis responsive to trimethoprim/sulfamethoxazole treatment in Japan. Neurol Neuroimmunol Neurolnflamm. 2015;2(5):e143

14. Wilson MR, Sample HA, Zorn KC, Arevalo S, Yu G, Neuhaus J, et al. Clinical metagenomic sequencing for diagnosis of meningitis and encephalitis. $\mathrm{N}$ Engl J Med. 2019;380:2327-40.

15. Salzberg SL, Breitwieser FP, Kumar A, Hao H, Burger P, Rodriguez FJ, et al. Next-generation sequencing in neuropathologic diagnosis of infections of the nervous system. Neurol Neuroimmunol Neurolnflamm. 2016;3(4):1-9.

16. Hu Z, Weng X, Xu C, Lin Y, Cheng C, Wei H, et al. Metagenomic nextgeneration sequencing as a diagnostic tool for toxoplasmic encephalitis. Ann Clin Microbiol Antimicrob. 2018;17:1-7. https://doi.org/10.1186/ s12941-018-0298-1.

17. Simner PJ, Miller S, Carroll KC. Understanding the promises and hurdles of metagenomic next-generation sequencing as a diagnostic tool for infectious diseases. Clin Infect Dis. 2018:66:778-88.

\section{Publisher's Note}

Springer Nature remains neutral with regard to jurisdictional claims in published maps and institutional affiliations.

Ready to submit your research? Choose BMC and benefit from

- fast, convenient online submission

- thorough peer review by experienced researchers in your field

- rapid publication on acceptance

- support for research data, including large and complex data types

- gold Open Access which fosters wider collaboration and increased citations

- maximum visibility for your research: over 100M website views per year

At BMC, research is always in progress.

Learn more biomedcentral.com/submissions 